Itinéraires Itinéraires

Littérature, textes, cultures

\title{
La traduction-guérison : un aperçu de cette métaphore à la lumière de quelques cas célèbres
}

Translation as Healing: An Overview of the Metaphor in the Light of Some

Famous Cases

\section{Aude A. Gwendoline}

\section{OpenEdition}

\section{Journals}

Édition électronique

URL : http://journals.openedition.org/itineraires/4930

DOI : 10.4000/itineraires.4930

ISSN : 2427-920X

\section{Éditeur}

Pléiade

\section{Référence électronique}

Aude A. Gwendoline, «La traduction-guérison : un aperçu de cette métaphore à la lumière de quelques cas célèbres », Itinéraires [En ligne], 2018-2 et 3 | 2019, mis en ligne le 20 février 2019, consulté le 17 juin 2019. URL : http://journals.openedition.org/itineraires/4930 ; DOI : 10.4000/itineraires.4930

Ce document a été généré automatiquement le 17 juin 2019.

\section{$(1) \Theta \Theta$}

Itinéraires est mis à disposition selon les termes de la licence Creative Commons Attribution - Pas d'Utilisation Commerciale - Pas de Modification 4.0 International. 


\section{La traduction-guérison : un aperçu de cette métaphore à la lumière de quelques cas célèbres}

Translation as Healing: An Overview of the Metaphor in the Light of Some

Famous Cases

Aude A. Gwendoline

«Traduire (transférer) : moins changer de langue que changer sa langue et, en elle, retrouver

l'étranger du langage.»

J.-B. Pontalis ${ }^{1}$

\section{Des métaphores du traduire}

1 Des métaphores pour tenter de décrire le traducteur dans sa tâche aux métaphores du processus à proprement parler que nous identifierons dans le présent article par le terme traduire, sans oublier la métaphore de la traduction appliquée de plus en plus fréquemment à tout transfert d'un système à un autre, ou encore le champ d'étude de la traduction des métaphores, les termes métaphore et traduction semblent intimement liés, ce que corroborent leurs étymologies respectives. Dans son article intitulé « Metaphors for the Translator » en effet, Michael Hanne évoque cette parenté étymologique :

Le terme anglais translation vient du latin translatus, participe passé du verbe transferre qui signifie "transporter", auquel fait également écho le terme anglais transfer. En traduction, cette étymologie suggère que le sens est transporté physiquement d'un pays et d'une culture, à travers une frontière - ou, ainsi que le formule Rosanna Warren, à travers l'abîme qui sépare une langue d'une autre (1989 : 3) - et déposé (inchangé) de l'autre côté.

Le terme anglais metaphor a des origines grecques, plutôt que latines : il vient des mots grecs meta (" au-delà ») et pherein (" porter, transporter »). Il implique donc lui aussi un "transport» mais un "transport» du contenu sémantique d'un domaine à un autre ${ }^{2}$. (Hanne $\left.2006: 208-209\right)$ 
2 Précisons d'ores et déjà que cet article portera sur le traduire littéraire, baptisé ainsi, dans un contraste voulu avec la traduction littéraire, pour mettre en valeur la pratique du traducteur, plutôt que les théories de la traduction, bien que celles-ci continuent d'être émaillées de métaphores - à visée justificative, explicative, voire, parfois, réductrice ainsi qu'on peut le lire au chapitre «De la naissance des théories - Métaphores et métonymies » d'Essais d'histoire de la traduction ${ }^{3}$ :

Sous la forte impulsion des théories linguistiques de la traduction nées au cours de la seconde moitié du Xx $x^{\mathrm{e}}$ siècle (de A. V. Fedorov à G. Mounin en passant par O. Kade et bien d'autres), les métaphores sur la traduction, de même que les démarches herméneutiques qui se servaient couramment d'elles, ont longtemps été qualifiées comme le substrat d'une pensée "préscientifique "; le jour viendrait où elles seraient évacuées du discours théorique, puis remplacées par un métalangage univoque et universel. Or, tout porte à croire que les métaphores sont au contraire une propriété durable des théories traductives, et que leur rôle continue aujourd'hui d'être analogue à celui qui était le leur dans le passé. (D'hulst 2014 : 45)

Parmi ces métaphores, citons les figures d'Hermès ${ }^{4}$ et d'Orphée ${ }^{5}$, mais aussi d'Écho, à laquelle fait référence Simona Elena Bonelli (2017 : 72), Écho, condamnée par Héra dans la mythologie grecque à avoir toujours le dernier mot, mais à ne jamais parler la première. Dans la pratique, cependant, on ne pourrait réduire le fait de traduire à un saint auquel le traducteur se vouerait exclusivement - celui du style et de la littérarité ou celui du sens et de la littéralité - de l'aube de sa tâche jusqu'au crépuscule de celle-ci. Traduire est une pratique polymorphe qui relève du paganisme et les dieux sont multiples au nom desquels le texte traduit est, parfois, sacrifié. Sacrifice du rythme et de la prosodie sur l'autel de la sémantique, ou inversement; sacrifice des jeux de mots par manque d'imagination adaptative, des références culturelles par souci de domestication (et d'économie de notes de bas de page), etc. Stigmate de la mauvaise presse accordée au traducteur, ce dernier reste encore largement invisible, en France, sur les pages de couverture des romans. Si on y prête quelque attention, on pourra découvrir son nom en quatrième de couverture. À cette «invisibilité » venutienne ${ }^{6} \mathrm{du}$ traducteur s'ajoute une panoplie de métaphores à connotation négative ${ }^{7}$ dont les plus courantes sont : la trahison, l'infidélité et même, le viol.

\section{Traduire, un acte violent}

Déjà, à travers les termes que Steiner employait dans Après Babel pour décrire le processus en quatre phases de la traduction - la « confiance », l'« agression », l'« incorporation » et le « retour du piston »-, on ressent cette violence. Selon Domenico Jervolino (2007: 80) : «[...] Steiner rappelle Hegel et Heidegger, mais aussi saint Jérôme qui utilise la métaphore du signifié capturé et conduit chez soi par le traducteur. »

Albert Bensoussan, dans son essai « La traduction littéraire : l'autre écriture », aborde ces concepts de trahison par opposition à la fidélité :

Le traducteur n'est pas un néant d'écriture, il n'en reste pas au «degré zéro de l'écriture »- pour reprendre la belle expression de Roland Barthes. Il ne met pas ses pas dans les traces de l'auteur pour une fallacieuse littéralité. Il existe comme créateur, ou plutôt, pour reprendre la terminologie de Claude Simon, comme «producteur de texte ». Et c'est justement cela qui fait problème. C'est même le vrai problème de la traduction. C'est bien pourquoi on a inventé le concept de fidélité qui est comme un carcan qui emprisonne le libre envol du traducteur et lui rogne les ailes. Un concept passible du tribunal ecclésiastique à la Renaissance : Luis de 
León, pour quelque liberté avec le texte canonique du Cantique des cantiques, fit cinq ans de prison à Salamanque, et à Paris Étienne Dolet, pour quelques contresens, finit sur l'échafaud. Nul ne l'a mieux vu aujourd'hui que l'écrivain franco-tchèque Milan Kundera qui y consacre les meilleures pages de son essai Les testaments trahis (Gallimard, Paris 1993). (Bensoussan 2000 : 262)

6 Les traducteurs littéraires eux-mêmes sont les premiers à s'accuser de trahison; en témoignent des ouvrages tels que Éloge de la trahison de Sylvie Durastanti, J'avoue que j'ai trahi - essai libre sur la traduction d'Albert Bensoussan (2005) ou encore Re-Belle et Infidèle la traduction comme pratique de réécriture au féminin de Susanne de Lotbinière-Harwood (1991), référence au célèbre essai Les Belles infidèles de Georges Mounin et à l'expression éponyme. Héritage du concept de plaisir du texte selon Roland Barthes, la métaphore de l'infidélité du traducteur s'inscrit également dans les théories de la matérialité du texte envisagé comme corps, un corps qui peut tout à fait être sexué, à l'instar de la traduction ; dès lors naît un plaisir subversif de la transgression, de la différence / différance dans la répétition.

7 Pour rester dans l'imagerie sexuelle ou sexuellement connotée, il importe de mentionner la métaphore du viol à laquelle René Ladmiral a recours dans un article publié dans le numéro de la revue Palimpsestes intitulé «L'étranger dans la langue » :

Dans les faits, c'est-à-dire dans la pratique traduisante réelle, les choses se règlent pour ainsi dire au coup par coup. Chaque item concret dont l'écriture fera problème, ce sera au traducteur (à moi, à vous, à quelqu'un) de prendre une décision, au niveau propre de la pratique, en arbitrant le conflit latent dont la norme de langue-cible est l'enjeu au terme d'un va-et-vient dialogique entre l'écoute de son propre sens de la langue (Sprachgefühl) et le risque à prendre d'un faire violence. $\mathrm{Au}$ reste, ce viol linguistique auquel il faudra que je me prête (et auquel déjà je m'apprête) n'est pas forcément désagréable, puisqu'aussi bien j'y serai à la fois le violeur et le (ou la) violé(e). En violant la langue, c'est en effet une part de moi-même que je viole moi-même ; car il est bien vrai que profondément ma langue m'habite. (Ladmiral 1993 : 31)

8 «Conflit », « violence », « viol » : le métalangage employé pour décrire le traduire est luimême synonyme d'un rapport de force qui se situe sur le plan linguistique. Henri Meschonnic abonde dans ce sens lorsqu'il écrit que «[1]e paradoxe est que, devant la littérature, ce n'est pas la littérature que vise la traduction, mais la langue » (1999: 84). Dans les pages suivantes de Poétique du traduire, il développe en ces termes :

La confrontation du traduire avec la littérature est donc la confrontation permanente de la langue au discours, des idéologies de la langue et de la littérature au fonctionnement historique de la littérature. C'est en cela que la littérature est une mise à l'épreuve du traduire, des idéologies du traducteur, de son passage ou non d'une linguistique spontanée implicite à une mise en question de ses pratiques. Cette mise à l'épreuve définit l'historicité du traduire, sa situation, qui s'inscrit dans sa traduction. La traduction littéraire montre et cache à la fois, par son écriture même, l'interaction de la théorie du langage et de la théorie de la littérature qui sont à l'œuvre dans le discours du traducteur. Que le praticien le veuille ou non, la théorie de la traduction est inévitable. Plus il refuse l'idée qu'il y a une théorie de la traduction, plus il renforce, par son refus même, la nécessité d'un examen des raisons qui le poussent à ce refus, du comment, du pourquoi et de l'historicité du traduire. Le rejet de la théorie fait partie de la théorie. (Meschonnic 1999: 85-86)

Il est un cas particulier où cette « confrontation permanente de la langue au discours, des idéologies de la langue et de la littérature au fonctionnement historique de la littérature " se complexifie encore davantage: lorsque les traducteurs littéraires sont eux-mêmes 
écrivains. Polyglottes, ils parlent la langue qu'ils traduisent, celle dans laquelle ils traduisent, mais aussi, et surtout, celle qu'ils pratiquent dans leur œuvre propre - leur langage ${ }^{8}$ à eux. Le rapport entre ces langues, leur «mise en réseau » selon l'expression d'Édouard Glissant, est au cœur même de leur pratique de la traduction :

Quand je parle de multilinguisme, [...] [c]e n'est pas une question de parler les langues, ce n'est pas le problème. [...] C'est plutôt la manière même de parler sa propre langue, de la parler fermée ou ouverte; de la parler dans l'ignorance de la présence des autres langues ou dans la prescience que les autres langues existent et qu'elles nous influencent même sans qu'on le sache. Ce n'est pas une question de science, de connaissance des langues, c'est une question d'imaginaire des langues. Et, par conséquent, ce n'est pas une question de juxtaposition des langues, mais de leur mise en réseau. (Glissant 1995 : 91)

Fait intéressant lorsqu'Édouard Glissant évoque la tradition de l'imaginaire des langues, il cite deux traducteurs-écrivains, le premier, Beckett, auto-traducteur comme Nancy Huston dont il sera ultérieurement question dans cet article, le second n'étant autre qu'Antonin Artaud, dont le cas fera tout de suite l'objet d'une analyse : «Il y a bien sûr une tradition en Occident de cette problématique de l'imaginaire des langues. Ce n'est pas d'aujourd'hui. Je crois que Beckett en serait un exemple. Artaud en est un autre: il a beaucoup déconstruit la langue » $(1995: 85)$.

\section{Traduire pour guérir : le cas Antonin Artaud}

11 Lorsqu'Antonin Artaud est interné à Rodez, il est soumis aux soins du psychiatre Gaston Ferdière, dont Emmanuel Venet écrit :

Il a vingt ans. Il veut croire qu'on peut impunément concilier médecine et littérature, nourrir sa langue du coudoiement avec la maladie et la mort comme le feindra Destouches, ou s'en arracher avec panache comme l'a fait Breton. [...] Ferdière atterrit d'abord à Villejuif. C'est là qu'il rencontre le verbe déstructuré, grandiose et hermétique des fous: la source même de toute poésie, l'endroit rêvé pour étancher enfin sa soif d'inouï et se lancer vraiment. (Venet $2006: 11,15)$

Ferdière, à l'avant-garde de l'art-thérapie, s'engage donc dans un vaste projet pour guérir son malade 9 auquel il réclame, entre deux séances d'électrochocs, des traductions, notamment de Lewis Carroll et Edgar Allan Poe, dans une tentative visant probablement davantage à faire renaître Artaud-écrivain (il ne produit alors plus d'œuvres) qu'à traiter la schizophrénie de son patient. Anne Tomiche explique les détails de cette pratique de la traduction chez Artaud :

[O]n ne peut parler d'Artaud comme on parlerait d'un poète qui, à l'instar de Baudelaire ou de Hölderlin, s'est reconnu dans l'activité de traducteur et l'a valorisée. Non seulement Artaud connaissait très mal l'anglais - il l'avouait luimême à Anaïs Nin au début des années 1930 - mais de plus, à cette époque, il donnait à la traduction une connotation très négative. [...]

Pourtant, à l'époque où, du 11 février 1943 au 25 mai 1946, il est interné à Rodez, «déporté en France» selon ses propres termes, Artaud se trouve confronté à plusieurs textes anglais qu'il traduit. Rodez: dernier asile psychiatrique dans une longue série commencée en septembre 1937 à l'arrivée au Havre lors du retour forcé d'Irlande, et qui fait suite à ceux de Sotteville-lès-Rouen, de Sainte-Anne à Paris, et de Ville-Evrard où il est enfermé de février 1939 à janvier 1943. À Rodez, d'abord à l'instigation du docteur Gaston Ferdière, directeur de l'asile et médecin traitant d'Artaud, ensuite de son plein gré, Artaud traduit ou adapte des textes, aidé dans son entreprise par l'abbé Henry Julien qui, de janvier 1941 à octobre 1944, y 
est aumônier et qui est angliciste (il enseigna au collège St Gabriel à St-Affrique dans l'Aveyron après son départ de Rodez). Julien rapporte :

Ce n'est qu'après un certain temps, sur le conseil du Dr Ferdière, qu'Artaud entrait vraiment en relation avec moi au titre d'anglicisant. Il semblait, alors, n'avoir qu'une connaissance assez quelconque de l'anglais et il ne pouvait traduire des textes sans aide. Lors de ces visites, il m'écoutait lire un texte et le traduire. Il reprenait la traduction, suggérait tel mot, telle tournure. [...] Il me rapportait les textes écrits de sa main, d'une écriture ample, régulière ${ }^{10}$. (Tomiche 1999 : 143-144)

Très vite, en juin 1944 selon Augusto Ponzio, Artaud «exprime la conviction qu'il est parvenu à sa traduction comme si elle était son œuvre propre, son commentaire personnel ${ }^{11}$ ». Dans le paragraphe " "Forcener le subjectile" ou comment renverser la figure de l'auteur en s'attaquant au texte-source » de son article, Jean-Yves Samacher avance la thèse suivante :

Nous faisons l'hypothèse que, dans la démarche scripturale propre à Artaud, le recours aux textes d'autres auteurs, qu'ils écrivent en français ou en langue étrangère, permet de faire surgir, littéralement, la «substantifique moelle » de l'écriture, grâce à cet acte particulier - exécuté au moyen de divers outils, procédés et techniques - qui consiste à «forcener le subjectile». Cette expression que Jacques Derrida emprunte au poète, lequel l'applique avant tout à sa manière de traiter ses œuvres plastiques et picturales, nous croyons possible et justifié de l'utiliser également à propos de son travail spécifique de traduction.

[...] En effet, l'attentat contre le support/modèle est l'un des seuls moyens, pour le sujet schizophrène, de se délivrer de la présence trop contraignante et persécutrice de l'Autre (la langue maternelle, l'auteur, les premiers traducteurs). Dans ce contexte, «forcener le subjectile » revient à s'attaquer non seulement aux corps imaginaire et symbolique du langage pour en faire ressortir la lettre, soit le corps réel de l'écriture, mais aussi à déformer et à défigurer le texte-source en vue d'affirmer l'authenticité, la singularité et la supériorité du traducteur-adaptateur. (Samacher $2011: 68-69$ )

Cette "traduction " - Artaud préférait employer l'expression "adaptation-variation » (Tomiche 2012: 47) - d'un passage de "Tèma con Variaziòni " (qu'il titre non pas "Thème avec variations » mais "Variations à propos d'un thème ») de Lewis Carroll est emblématique de ce retour à soi de l'écrivain, par le détour de la traduction et la définition d'une nouvelle poétique de la traduction ${ }^{12}$, et de la réappropriation de son statut d'auteur par Antonin Artaud qui va jusqu'à accuser Carroll de plagiat par un mouvement inversé, un jeu de miroir dans lequel le traducteur ne traduit plus, il est traduit, l'auteur du texte original devenant alors, lui, plagiaire :

To glad me with his soft black eye

My son comes trotting home from school;

He's had a fight, but can't tell why-

He always was a little fool!

lorsque je vois venir à moi avec un œil poché et noir

mon fils à l'heure de la sortie des classes

s'étant battu contre qui contre quoi

et ne sachant trop dire pourquoi,

j'ai l'impression de me voir moi

en bataille devant ma glace

contre mon propre désespoir.

15 Soulignons le glissement du « moi » objet en anglais $(m e)$ au «moi » sujet en français (je), subjectivisation appuyée par la pronominalisation à outrance à la première personne du singulier (à moi ; moi ; ma; mon propre). Au moyen de cette "glace ", Artaud introduit non seulement son «moi» dans le poème, il réintroduit également la figure du miroir, 
absente de l'original, laquelle est à relier au stade du miroir psychanalytique ou étape par excellence de subjectivisation :

La figure du miroir est absente de "Tèma con Variaziòni ». Par contre, on connaît son importance dans les aventures d'Alice, en particulier dans Through the LookingGlass où elle est inscrite dans le titre et où elle est le pivot du « monde à l'envers » et du « système général d'inversions » qui caractérisent l'autre côté du miroir. [...] Artaud s'empare de la figure du miroir pour l'introduire dans sa traduction. Ce faisant, s'accomplit un triple mouvement : mouvement de déplacement intertextuel (la figure du miroir est déplacée de Through the Looking-Glass vers " Variations à propos d'un thème "); mouvement de superscription (puisque dans la traduction d'Artaud la figure du miroir vient se surinscrire sur le texte de Carroll); mouvement de répétition (le miroir est une figure du redoublement, donc de la répétition).

La figure du miroir apparaît tant dans le texte en prose que dans le poème. Dès le début de son texte en prose, Artaud qui affirme avoir voulu, dans son " adaptationvariation », «rejoindre l'auteur en esprit », commente le texte de Lewis Carroll en disant: «Lewis Carroll a vu son moi comme dans une glace ». L'écriture poétique - celle de Carroll qu'Artaud cherche à "rejoindre en esprit »- a donc fonction de miroir. Qui plus est, comme l'écriture, la lecture (celle de Carroll par Artaud, celle de tous les poètes) est une confrontation au miroir : « lire l'œuvre d'un poète c'est avant tout lire au travers. Car toute œuvre écrite est une glace » [...]. Dans ce textemiroir, la relation spéculaire du sujet poète à son image rejoue une sorte de «stade du miroir ", au cours duquel le sujet se constitue sur le double mode de la méconnaissance - reconnaissance et aliénation simultanées - et de l'agressivité. (Tomiche 2012 : 67-68)

Les limites des compétences langagières d'Artaud en anglais lui rouvrent les portes de la créativité dans la mesure où le "concept de fidélité qui est comme un carcan qui emprisonne le libre envol du traducteur et lui rogne les ailes » pour reprendre les termes de Bensoussan, cité plus haut, disparaît. Comme l'écrit Anne Tomiche :

Les traductions de Rodez marquent ainsi le moment et le mouvement d'un retour à la poésie, mouvement à partir duquel s'élabore une poétique propre à Artaud, qui est une poétique de la voix, du rythme et de la scansion, qui prendra de plus en plus d'ampleur à la sortie de Rodez. La traduction, en tant que confrontation à la langue étrangère et au texte étranger, constitue alors, dans les termes de Camille Dumoulié, une "voie de traverse, une stratégie du détour et de l'oblique», qui permet, « par le biais des autres, de biais, [de] "se refaire" ». (Tomiche $2012: 20$ )

\section{Guérir par le traduire : Nancy Huston, Louis Wolfson et Anna 0.}

17 Après avoir écrit ses premiers romans en français - seconde langue de cette écrivaine anglo-canadienne devenue française -, Nancy Huston a rejoint le panthéon de ces autotraducteurs célèbres (Beckett, Green, Nabokov) en 1987 lorsqu'elle a traduit en anglais son deuxième roman, Histoire d'Omaya. Il faudra attendre 1996 pour qu'elle traduise Les Variations Goldberg (1981) en anglais. Susan Bassnett s'est entretenue avec l'écrivaine à l'identité linguistique double, ou « bidentité » :

Huston a dressé un parallèle intrigant et puissant entre l'auto-traducteur s'efforçant d'exister dans deux langues distinctes et une enfant prise dans l'engrenage du divorce de ses parents et contrainte de passer de son père à sa mère en courant :

essayant d'expliquer maman à papa et papa à maman, écoutez-vous tous les deux, ça n'en a peut-être pas l'air, mais en fait, vous dites exactement la même chose, 
écoutez écoutez, vous êtes compatibles, restez ensemble, ne vous séparez pas, ne volez pas en éclats, ne nous détruisez pas tous en détruisant votre mariage, (...) pourquoi un tel fossé entre anglophones et francophones, les choses importantes sont les mêmes dans nos vies, n'est-ce pas? (communication personnelle)

Pourtant, au terme du processus, Huston reconnaît qu'elle ressent une certaine satisfaction à pouvoir raconter les mêmes histoires dans deux langues : « comme si, d'une certaine façon, j'avais réussi à prouver que je ne suis pas schizophrène, pas folle. Parce qu'en fin de compte, la même personne dans les deux langues " (communication personnelle). Pour Nancy Huston, l'auto-traduction est difficile et douloureuse parce qu'au premier abord, elle semble exposer des écarts entre les langues, faire surgir le spectre d'un esprit divisé et d'un monde divisé, mais une fois la traduction achevée, les écarts sont comblés, le processus est devenu celui d'une guérison et l'auto-traducteur n'est plus tiraillé entre deux langues, mais capable d'exister pleinement dans les deux. Huston reconnaît que, d'une façon ou d'une autre, le fossé entre ses deux moi linguistiques a été guéri par l'intermédiaire de la traduction $^{13}$. (2013: 15-16, notre traduction)

Nancy Huston s'auto-guérit en s'auto-traduisant et sa démarche n'est pas sans rappeler la traversée du miroir opérée par Artaud traducteur-devenu-auteur des textes de Carroll dans la mesure où l'écrivaine, après s'être auto-traduite vers sa langue maternelle, donc par l'intermédiaire d'un détour par l'étranger, à l'instar d'Artaud, a désormais recours à une technique d'écriture multilingue simultanée puisqu'elle passe de l'anglais au français et du français à l'anglais au sein d'un même roman, lors de la phase de création. Ainsi, elle brouille les pistes entre Nancy l'écrivaine et Nancy la traductrice de sorte que l'autotraduction devient le lieu de la réconciliation entre Nancy l'anglophone et Nancy la francophone, et un gage de guérison du moi potentiellement schizophrène.

Louis Wolfson, schizophrène lui aussi et psychotique, a recours à la traduction de l'anglais, sa langue maternelle. Dans la préface de son ouvrage Le Schizo et les langues intitulée « Schizologie », Gilles Deleuze explique la démarche de l'écrivain (Wolfson [1970] $2013: 5-6)$ :

L'auteur est américain, mais le livre est écrit en français, pour des raisons qui paraîtront tout de suite évidentes. Car ce que fait l'étudiant, c'est traduire suivant certaines règles. Son procédé scientifique est le suivant: un mot de la langue maternelle étant donné, trouver un mot étranger de sens similaire, mais aussi ayant des sons ou des phonèmes communs (de préférence en français, allemand, russe ou hébreu, les quatre langues principalement étudiées par l'auteur). Une phrase maternelle quelconque sera donc analysée dans ses éléments et mouvements phonétiques, pour être convertie le plus vite possible en une phrase d'une ou plusieurs langues étrangères à la fois, qui ne lui ressemble pas seulement en sens, mais en son. Le plus vite possible... mais, comme la transformation peut faire intervenir plusieurs états intermédiaires, elle sera d'autant plus féconde qu'elle mettra en jeu des règles phonétiques générales applicables à d'autres transformations, couvrant ainsi le plus d'espace linguistique possible (même au prix de fautes de syntaxe ou d'inexactitudes de sens).

Il y a dans l'acte de traduire sur la base d'une musique du phonème plutôt que sur celle d'une tyrannie de la syntaxe, de la grammaire ou de la sémantique, un affranchissement vis-à-vis de la langue malade, à savoir la langue maternelle, et de la maladie. La traduction entre les langues lui sert à la fois d'échappatoire et de survie dans sa langue propre, celle qu'il habite. Selon Jean-Marie Gustave Le Clézio, « [1]a seule recherche, pour l'étudiant malade mental, sera la recherche de cette possibilité de communication, par d'autres moyens que celui du langage habituel » (2009: 46-47). 
21 Enfin, abordons le cas d'Anna O., patiente hystérique de Freud, qui «souffrait d'une maladie langagière, qui lui fit perdre dans un premier temps sa langue maternelle ", l'allemand, ainsi que l'explique Catherine Mavrikakis (1998: 73) :

Toutes les langues disparaissent au profit de la langue anglaise. Anna O. devient interprète, traductrice simultanée vers l'anglais, dans une folie langagière incompréhensible. On pourrait dire qu'Anna $\mathrm{O}$. se transforme en machine à tout déplacer, à tout traduire vers l'anglais et même les gens de son entourage se retrouvent à parler aussi, et ce, malgré eux, la langue de Shakespeare, très littéralement ici. L'hystérie devient alors un véritable logiciel de traduction.

Or Fräulein Anna O. (comme l'appelle Freud) va se mettre, en anglais, à raconter sa vie. C'est elle qui va donner au procédé de la cure le nom de «talking cure » ou encore de "chimney sweeping ». Les symptômes d'Anna vont alors disparaître, avec une histoire sur une méchante dame de compagnie anglaise et une autre, très importante, qui porte sur un vers enfantin anglais qu'elle récitait, après une hallucination sexuelle et après avoir perdu la parole, au chevet de son père. "Immédiatement après ce récit elle s'exprima en allemand et se trouva dès lors débarrassée des troubles qui l'avaient affectée auparavant» (Freud, 1973, p. 30). Pour Freud, le processus de la cure se trouve terminé lorsque les troubles qui affectent Anna disparaissent, mais aussi et surtout quand celle-ci est capable de retourner à la langue allemande et de stopper la machine à traduire qu'elle était devenue temporairement. (1998: 74-75)

En conclusion de son article, Catherine Mavrikakis parle de l'anglais, la langue de traduction d'Anna O., comme d'une «langue de passage vers la santé » (ibid.: 91, nous soulignons), autrement dit la traduction linguistique permet le transfert, le « passage » ou encore, la traduction d'un état maladif en retour effectif à la santé.

\section{Conclusion}

23 La traduction a longtemps été enfermée dans une sphère négative où abondaient les métaphores péjoratives, voire violentes, pour la décrire. Elle reste, encore aujourd'hui, dans de nombreux cas, cantonnée à un statut de sous-art, comparativement à la création " pure » de l'écrivain. Pourtant, les cas plus ou moins «cliniques » présentés dans cet article posent question: que savons-nous, à l'aube du xxi ${ }^{\mathrm{e}}$ siècle de la traduction ? Les avancées des neurosciences, les recherches scientifiques sur le cerveau du traducteur, sur ses mirror neurons (concept par ailleurs lui-même contesté), sont autant de pistes de réflexion invitant à considérer enfin la traduction non plus du point de vue de l'art, mais de celui de la science.

Et si le détour par l'étranger qu'est la langue étrangère était une voie de guérison potentielle pour le schizophrène dont la langue est autant malade qu'il est malade de sa langue et dans sa langue?

Traduire pour guérir, guérir en traduisant - ces expressions appliquées à Artaud, Huston, Wolfson et Anna $O$. sont par ailleurs transposables à l'approche scientifique du psychanalyste qui n'effectue pas autre chose que des traductions, lors de l'analyse ${ }^{14}$. Les métaphores du traduire ont autant à dire que le traduire comme métaphore.

Les recherches traductologiques actuelles semblent en outre se centrer sur l'étude du sujet qu'est le traducteur, davantage que sur l'analyse de l'objet que constitue l'une ou l'autre traduction (encore trop souvent perçue comme étant en-deçà - et non pas audelà - de la création originale). Cette tendance s'intègre logiquement à l'approche de chercheurs qui s'intéressent moins à une analyse "figée " d'un texte d'arrivée qu'au 
processus en jeu en ce qu'il reflète non seulement la pratique d'un professionnel, mais aussi et surtout son rapport au langage. C'est sur ce rapport qu'il importe de se pencher afin de mesurer la portée de la traductologie, abordée sous l'angle d'une analyse de la pratique. Pour les sujets dont il était question ici, traduire ou pratiquer une langue autre coïncide avec une renaissance en tant qu'auteur (Artaud), une réconciliation avec soi (Huston), une forme d'auto-traitement par le langage (Wolfson) ou une cure analytique (Anna 0.). Ces exemples probants sont autant d'invitations à explorer le potentiel du multilinguisme à influer (positivement) sur des identités et des singularités propres.

\section{BIBLIOGRAPHIE}

Bassnett, Susan, 2013, «The Self-Translator as Rewriter », dans A. Cordingley (dir.), Self-

Translation: Brokering Originality in Hybrid Culture, Londres, Bloomsbury, p. 13-25.

Bensoussan, Albert, 2000, «La traduction littéraire : l'autre écriture », dans S. Petrilli (dir.), La traduzione, Rome, Meltemi, p. 259-267.

Bensoussan, Albert, 2005, J'avoue que j'ai trahi. Essai libre sur la traduction, Paris, L'Harmattan.

Bonelli, Simona Elena, 2017, « Hybridité du corps-texte dans l'autobiographie de Princesa », dans A. Nouss, C. Pinçonnat et F. Rinner (dir.), Littératures migrantes et traduction, Aix-en-Provence, Presses universitaires de Provence, p. 65-74.

D’hulst, Lieven, 2014, Essais d'histoire de la traduction : avatars de Janus, Paris, Garnier.

Durastanti, Sylvie, 2002, Éloge de la trahison : notes du traducteur, Paris, Le passage.

Glissant, Édouard, 1995, Introduction à une poétique du divers, Montréal, Presses de l'Université de Montréal.

Glissant, Édouard, 2010, L'imaginaire des langues - Entretiens avec Lise Gauvin (1991-2009), Paris, Gallimard.

Hanne, Michael, 2006, « Metaphors for the Translator », dans S. Bassnett et P. Bush (dir.), The Translator as Writer, New York, Continuum, p. 208-224.

Jervolino, Domenico, 2007, Ricœur. Herméneutique et traduction, Paris, Ellipses.

Ladmiral, Jean-René, 1993, « La langue violée ? ", Palimpsestes, n 6, [En ligne], https:// journals.openedition.org/palimpsestes/754, consulté le 20 janvier 2018.

DOI : $10.4000 /$ palimpsestes.754

Le Blanc, Charles, 2009, Le Complexe d'Hermès, Ottawa, Presses de l'Université d'Ottawa.

Le Clézio, Jean-Marie Gustave, 2009, « La tour de Babil », dans J.-B. Pontalis, J.M.G. Le Clézio, P. Auster, P. Aulagnier, M. Dorra, M. Foucault, P. Alferi et F. Cusset, Dossier Wolfson ou L'affaire du Schizo et les langues, Paris, Gallimard.

Lotbinière-Harwood, Susanne de, 1991, Re-belle et infidèle : la traduction comme pratique de réécriture au féminin / The Body Bilingual: Translation as a Rewriting in the Feminine, Montréal, Toronto, Les éditions du remue-ménage, Women's Press. 
Mavrikakis, Catherine, 1996, La Mauvaise Langue, Seyssel, Champ Vallon.

Mavrikakis, Catherine, 1998, « L'hystérique face aux symptômes de la traduction », TTR: traduction, terminologie, rédaction, vol. 11, n 2, p. 73-93.

Meschonnic, Henri, 1999, Poétique du traduire, Lagrasse, Verdier.

Ponzio, Augusto, 2007, « Translation and the Literary Text », TTR : traduction, terminologie, rédaction, vol. 20, n² 2, p. 89-119.

Raimondo, Riccardo, 2016, « Orphée contre Hermès : traduction, herméneutique et imaginaire (esquisses) », Meta, vol. 61, nº 3, p. 650-674, [En ligne], https://id.erudit.org/iderudit/1039223ar, consulté le 20 javnier 2019.

DOI : 10.7202/1039223ar

Samacher, Jean-Yves, 2011, « Corps et traduction dans les textes d'Antonin Artaud », Psychologie Clinique, $\mathrm{n}^{\circ} 32$, p. 61-74.

Tomiche, Anne, 1999, « Traduction, transformation, appropriation : Antonin Artaud face à Lewis Carroll », dans C. Foucrier et D. Mortier (dir.), Frontières et Passages, Rouen, Publications de l'Université de Rouen, p. 143-156.

Tomiche, Anne, 2012, "L'intraduisible dont je suis fait », Artaud et les avant-gardes occidentales, Paris, Le Manuscrit.

Venet, Emmanuel, 2006, Ferdière, psychiatre d'Antonin Artaud, Lagrasse, Éditions Verdier.

Verger, Mathias, 2015, « Antonin Artaud et l'imaginaire de la traduction », Carnets de Chaminadour , no 4, p. 61-85.

Waisman, Sergio, 2005, Borges and Translation: The Irreverence of the Periphery, Cranbury, Associated University Press.

Wolfson, Louis, [1970] 2013, Le Schizo et les Langues, Paris, Gallimard.

Woodsworth, Judith, 1988, « Traducteurs et écrivains : vers une redéfinition de la traduction littéraire », TTR : traduction, terminologie, rédaction, vol. 1, n 1, p. 115-125.

\section{NOTES}

1. Cité dans Tomiche (2012: 11 ; source : Jean-Bernard Pontalis, L'Écrit du Temps, $\mathrm{n}^{\circ} 7$, été 1984 , p. 75).

2. "The English word translation derives from the Latin translatus, past participle of the verb transferre, meaning 'to carry across', which is echoed also in the English transfer. In translation, this etymology suggests, meaning is picked up bodily from one country and culture, transported across a frontier - or, as Rosanna Warren expresses it, across the chasm which separates one language from another (1989: 3) - and deposited (unaltered) on the other side. The English term metaphor has Greek, rather than Latin, origins, coming from the Greek terms meta ('beyond') and pherein ('to bear or to carry'). So it too involves 'carrying across', but a 'carrying across' of semantic meaning from one domain to another. »

3. Mentionnons également les nombreuses métaphores répertoriées dans l'article de Judith Woodsworth « Traducteurs et écrivains : vers une redéfinition de la traduction littéraire » (1988) qu'elle scinde en deux groupes - les métaphores à connotation négative et celles à connotation positive.

4. Charles Le Blanc y a consacré tout un ouvrage - Le complexe d'Hermès (2009) - qui s'inscrit dans le champ de la philosophie de la traduction. 
5. Riccardo Raimondo résume ainsi les figures métaphoriques qu'il emploie: "On pourrait synthétiser les approches de la traduction à l'aide de deux figures mythiques opposées : Hermès et Orphée. Le premier est métaphore d'une traduction herméneutique qu'on pourrait dire sourcière, visant à transposer le sens. De l'autre côté demeure Orphée, personnage qui tâtonne dans l'obscurité des formes et qui est métaphore d'une traduction qu'on pourrait qualifier de cibliste, visant le style. » Dans un troisième temps, il propose de recourir à la figure d'Apollon pour définir une voie de traduction alternative, "voie mystérieuse de récréation et révélation » selon ses termes (Raimondo $2016: 650$ ).

6. Lawrence Venuti, traductologue américain, est à l'origine du concept d'invisibilité du traducteur.

7. Notons que des écrivains et traducteurs tels que Borges ont pris un parti délibérément aux antipodes de ces critiques et préjugés vis-à-vis du traducteur en lui conférant une certaine supériorité par rapport à l'écrivain de l'œuvre originale traduite, ainsi qu'Albert Bensoussan le rappelle (2000: 259) : «Le grand écrivain argentin Jorge Luis Borges, friand de paradoxes, n'hésitait pas à considérer la traduction littéraire comme l'aboutissement de l'écriture, comme quelque chose de plus achevé que l'écriture de la langue originale: "Le métier de traducteur, écrivait-il, est peut-être plus subtil, plus civilisé que celui d'écrivain : il est évident que le traducteur vient après l'écrivain. La traduction est une étape plus avancée." "

8. Au chapitre «Passages de langues et territoires du roman » de L'imaginaire des langues, Édouard Glissant déclare (2010 : 105) : «Je ne crois pas que le fait de connaître plusieurs langues donne une prédisposition particulière à l'écriture. Ce qui donne une prédisposition particulière à l'écriture, c'est le fait d'avoir une prédisposition particulière à un langage, que ce langage soit dans une langue ou dans plusieurs langues. »

9. «Dans “J'ai soigné Antonin Artaud", publié dans La Tour de feu bien après la mort d'Artaud, Ferdière écrira : "J'avais rendu Artaud à la création artistique et poétique [...] sans moi Artaud serait mort dans la stérilité et le marasme [...]. Le résultat que j’ai obtenu [...] était dû dans une large mesure à l'Art-thérapie. [...] La main d'Artaud a dû réapprendre à écrire, grâce à la correspondance de plus en plus nombreuse qu'il entretenait avec ses amis (et, au début, il fallait le forcer à une réponse, même courte et encombrée de formules toutes faites), grâce surtout aux traductions que je lui demandais amicalement." On pousse donc bien Artaud à écrire. Et, dans les termes même du discours médical officiel, la remise à l'écriture passe par le chemin de la traduction: c'est la confrontation à l'étranger qui permet de revenir à soi » (Tomiche 2012 : 16-17).

10. Henri Julien, «J'étais donc l'aumônier... », La Tour du Feu, $\mathrm{n}^{\circ} 125$, repris dans le $\mathrm{n}^{\circ} 136$, décembre 1977, numéro special Artaud. La santé des poètes, p. 43 (cité par Anne Tomiche).

11. "Artaud very soon (June 1944) expressed the conviction that he had reached his translation as though it were his own original work and personal comment» (Ponzio 2007: 101, notre traduction).

12. "Antonin Artaud n'écrit pas en français. Il écrit dans le français, comme dans une matière en fusion, c'est-à-dire dans une langue française qui a conscience de sa malléabilité dans le temps et l'espace, conscience aussi de sa puissance de métamorphose. Processus instable en transformation plutôt que nomenclature rigide, le vocabulaire d'Antonin Artaud vient dire la vérité de la langue, vérité d'une langue toujours déjà et toujours encore en traduction » (Verger $2015: 61)$.

13. "Huston has drawn an intriguing and powerful parallel between the self-translator struggling to exist in two distinct languages and a child caught up in her parents' divorce, running back and forth between father and mother: "trying to explain mummy to daddy and daddy to mummy, listen to you guys, it may not sound like it but in fact you're saying exactly the same thing, listen listen, you're compatible, stay together, don't break up, don't fly apart, don't destroy us all by destroying your marriage, (...) why such a deep riff between anglophones and 
francophones, the important things are the same in our lives, aren't they? (personal communication)" Yet by the end of the process, Huston acknowledges that she feels a sense of satisfaction at being able to tell the same stories in two languages: 'as if that somehow proved that I'm not schizophrenic, not crazy. Because ultimately the same person in both languages' (personal communication). To Nancy Huston, self-translation is difficult and painful because it appears at first to expose gaps between languages, to raise the spectre of a divided mind and of a divided world, but when the translation is completed, the gaps are closed, the process has become of a healing one and the self-translator is no longer caught between languages but able to exist fully in both. Huston acknowledges that, somehow, the split between her two language selves has been healed through translation. »

14. Si on est loin, dans le travail qu'Artaud effectue à partir de Carroll, des définitions linguistiques de la traduction, on retrouve par contre le sens que Freud donne au terme d' Übersetzung tout au long de son œuvre et dans des contextes variés, quand il associe à la traduction le processus de conversion hystérique, le processus de formation des rêves, le fonctionnement de l'appareil psychique, le transfert, et enfin le travail de l'analyste lui-même. Quel que soit le contexte, l'analyse freudienne souligne la dimension énergétique du mouvement traducteur. Dans le cas du rêve, en particulier, la traduction constitue le " travail du rêve » dont les opérations sont la condensation, le déplacement et la figuration. «[...] Que ce soit dans le contexte du symptôme de conversion hystérique, dans celui du rêve, ou dans celui du passage à la conscience, il n'y a pas, pour Freud, d'un côté un original (un conflit psychique, le contenu latent d'un rêve, une représentation inconsciente) et, de l'autre, sa version traduite dérivée (en symptôme, contenu manifeste d'un rêve, représentation consciente). Il n'y a qu'une multiplicité de scènes traumatisantes ou de représentations refoulées, qui n'acquièrent leur sens que par un effet d'après-coup : il n'y a donc qu'une série de traductions et de re-traductions sans qu'il y ait accès direct à un original ; la traduction (symptôme de conversion ou rêve manifeste) n'est pas la transcription d'un original qui serait présent ailleurs, enfoui dans l'inconscient, et qu'il faudrait désenfouir. La traduction est, dans sa secondarité même, originaire et irréductible » (Tomiche 1999 : 149-150).

\section{RÉSUMÉS}

Traduire, trahir, tromper, faire des infidélités, violer la langue même - autant de métaphores d'une violence faite au texte littéraire par le traducteur lors du processus traductif. Pourtant, les cas où traduire a eu un effet salutaire existent, dans la littérature, mais aussi en psychiatrie et en psychanalyse. Que nous apprend l'expérience d'Antonin Artaud, de Nancy Huston, de Louis Wolfson ou d'Anna $\mathrm{O}$. sur la portée de l'acte consistant à traduire pour guérir et quelles portes ouvrent ces passerelles vers la guérison à la traduction envisagée comme art-thérapie ou comme pratique scientifique aux propriétés curatives?

Translating, betraying, deceiving, cheating, being unfaithful, even violating the language-these are all metaphors of violence against the literary text by the translator during the translation process. Nevertheless, there are cases where translating had a beneficial effect, in literature, but also in psychiatry and psychoanalysis. What do the experiences of Antonin Artaud, Nancy Huston, Louis Wolfson and Anna O. tell us about the possibility of healing through translating? 
What is the future of translation as an art therapy or as a scientific practice with healing properties?

INDEX

Mots-clés : traduction, guérison, métaphore, écrivains-traducteurs, Artaud (Antonin)

Keywords : translation, healing, metaphor, translators writers, Artaud (Antonin)

\section{AUTEUR}

AUDE A. GWENDOLINE

École de traduction, Université York, Campus Glendon, Toronto, Canada 